\title{
TENSOR PRODUCTS OF BANACH ALGEBRAS WITH INVOLUTION
}

\author{
BY \\ KJELD B. LAURSEN $\left({ }^{1}\right)$
}

1. Introduction. The present paper deals with tensor products of Banach algebras with involution. In a sense this paper may be considered an investigation of the extent to which certain properties of Banach-*-algebras are passed on to their tensor products.

In $\S \S 2$ and 3 we present much of the background material needed throughout the paper. In $\$ 2$ we gather some facts from the general representation theory and we introduce the notions of the spectrum and the enveloping algebra of a Banach-*algebra. In $\S 3$ we turn our attention to tensor products, specifically the problem of norming a given tensor product.

$\$ 4$ deals with the enveloping algebra of a tensor product. We show that with certain conditions on two Banach-*-algebras and on the norm norming their tensor product the enveloping algebra of their tensor product can be identified with a tensor product of their respective enveloping algebras. The main result of this section generalizes a theorem by Okayasu [16, Theorem 3].

In the following section we relate the spectrum of a tensor product to the product of the spectra of the Banach-*-algebras in question. Without restrictions on these algebras the latter product will be a subset of the former, but if one of the algebras is of type I, then the two sets can be identified. $\S 5$ is to a large extent a natural extension of work done by Wulfsohn [25], although connections also exist with results due to Okayasu and Takesaki [16], [17], to Gelbaum [4], [5], [6] and Gil de Lamadrid [7], [8].

On the basis of the theory developed up to this point we consider in $\$ 6$ the concept of $*$-semisimplicity. We show that a tensor product of $*$-semisimple Banach-*algebras is $*$-semisimple if and only if a variation of a condition of approximation introduced by Grothendieck [9] is satisfied.

The theory developed in this paper finds a natural application in the study of certain vector valued group algebras. The last section deals with this. We consider group algebras of functions from a compact $T_{2}$ group to Banach-*-algebras. The enveloping algebra and the spectra of such algebras are identified. Moreover, it is shown that $*$-semisimplicity of the range algebra is passed on to the group algebra

Presented, in part, to the Society, April 15, 1967 under the title Tensor products of Banach-*algebras; received by the editors August 17, 1967 and, in revised form, November 28, 1967.

( $\left.{ }^{1}\right)$ This paper is an extension of parts of the author's Ph.D. Thesis written at the University of Minnesota under Professor Gil de Lamadrid. The work was supported in part by the National Science Foundation. 
in question. This section can be considered a continuation of work done by Spicer [20], [21].

2. Background. Representations, the spectrum, the enveloping algebra. In this section we collect much of the theory of algebras with involution which will be needed later. We remark at the outset that we shall always use single bars $|\cdot|$ to denote the norm in a normed linear space. Moreover, to avoid confusion with the commutant of a set we shall use the notation $E_{*}$ to denote the dual space of a normed linear space $E$.

2.1. A representation $\mu$ (sometimes $*$-representation) of a $*$-algebra $A$ is an involution-preserving homomorphism of $A$ into the $C^{*}$-algebra $L\left(H_{\mu}\right)$ of all bounded linear operators on some Hilbert space. If the representation is an isomorphism, i.e. 1-1, we say it is faithful.

2.2. A representation $\mu$ on a Hilbert space $H_{\mu}$ is topologically irreducible if $H_{\mu}$ and $\{0\}$ are the only closed subspaces of $H_{\mu}$ left invariant by $\mu(A)$.

The von Neumann algebra (or $w^{*}$-algebra) generated by a set $B \subset L(H)$ will be denoted by $w^{*}(B)$ and the commutant of $B$ will be denoted by $B^{\prime}$. We also use the notation

$$
C_{H}=\{\lambda I ; \lambda \in C\} \subset L(H) .
$$

Here $C$ is the complex field and $I$ is the identity operator on $H$.

Using this notation we can list some characterizations of topological irreducibility; most of these are proved in [3, Proposition 2.3.1].

Proposition 2.3. If $A$ is a $*$-algebra and $\mu$ a representation of $A$ in a Hilbert space $H_{\mu}$ then the following conditions are equivalent:

(i) $\mu$ is topologically irreduc!ble.

(ii) $[\mu(A)]^{\prime}=C_{H_{\mu}}$.

(iii) Every nonzero vector $h$ in $H_{\mu}$ is cyclic for $\mu$, i.e. $H_{\mu}=\{\mu(x) h ; x \in A\}^{-}$where denotes norm closure.

(iv) $[\mu(A)]^{\prime \prime}=L\left(H_{\mu}\right)$.

(v) $w^{*}[\mu(A)]=L\left(H_{\mu}\right)$.

2.4. The spectrum of a *-algebra $A$, denoted by $\hat{A}$ or $A^{\wedge}$, is the set of equivalence classes (relative to the unitary equivalence $\cong$ (see for example [3, p. 26])) of topologically irreducible representations on Hilbert space. If $\mu$ is topologically irreducible then $\bar{\mu}$ or $\mu^{\wedge}$ will denote the element of $\hat{A}$ to which $\mu$ belongs.

2.5. A continuous linear functional $f$ on a normed *-algebra $A$ is positive if $f\left(x^{*} x\right) \geqq 0$ for all $x \in A$. $f$ is a state if $|f|=1$ and pure (or indecomposable) if for any positive continuous linear functional $g$ such that $f-g$ is positive it follows that $g$ is a multiple of $f$.

2.6. An algebra $A$ with involution is of type $\mathrm{I}$ if all representations of $A$ are of type I (see [3, Definition 5.4.2]). 
2.7. If we denote by $P(A)$ the set of pure states on a Banach-*-algebra $A$ with approximate identity then there is a natural mapping $\delta: P(A) \rightarrow \hat{A}$. $\delta$ is onto since if $\pi \in \bar{\pi} \in \hat{A}$ and $p$ is a pure state associated with $\pi$ (i.e. $p(x)=(\pi(x) \xi \mid \xi)$ for some $\left.\xi \in H_{\pi}\right)$ then the representation generated by $p$ is equivalent to $\pi[3,2.4 .6] . P(A)$ is a subset of the unit ball in the dual space of $A$ and thus has a natural topology, the relative weak-*-topology. We topologize $\hat{A}$ by giving it the quotient topology relative to $\delta$, i.e. the strongest topology on $\hat{A}$ with respect to which $\delta$ is continuous. If $A$ is a $C^{*}$-algebra then this definition yields the same topology as the one defined in terms of the Jacobson topology on the set of primitive ideals (see [3, p. 60 and $\S 3.4])$. In 2.9 we shall show that $\delta$ is open.

2.8. Let $A$ be a Banach-*-algebra and let $R$ be the set of representations of $A$. For each $x \in A$ define $|x|^{\prime}=\sup _{\pi \in R}|\pi(x)|$.

Then $|\cdot|^{\prime}$ is a seminorm on $A$ with the properties (i) $|x y|^{\prime} \leqq|x|^{\prime}|y|^{\prime}$, (ii) $\left|x^{*}\right|^{\prime}$ $=|x|^{\prime}$, and (iii) $\left|x^{*} x\right|^{\prime}=|x|^{\prime 2}$ for all $x, y \in A$. The set

$$
R^{*}=\left\{x \in A ;|x|^{\prime}=0\right\}
$$

is called the *-radical and will be studied in $\S 6$. Clearly, the completion of $A / R^{*}$ with respect to the quotient norm of $|\cdot|^{\prime}$ is a $C^{*}$-algebra. This $C^{*}$-algebra is the enveloping algebra of $A$ and is denoted by $C^{*}(A)$. The natural mapping $\varepsilon: A \rightarrow C^{*}(A)$ is called the enveloping mapping.

2.9. Let $A$ be a Banach-*-algebra with approximate identity. By $[3,2.7 .4]$ there is a 1-1 mapping $S: \hat{A} \rightarrow\left[C^{*}(A)\right]^{\wedge}$. Moreover, if $\varepsilon_{*}:\left(C^{*}(A)\right)_{*} \rightarrow A_{*}$ is the adjoint mapping of the enveloping mapping $\varepsilon$, then the restriction $Q=\left.\varepsilon_{*}\right|_{P\left(C^{*}(A)\right)}$ is a homeomorphism. Here we shall show that $S$ is a homeomorphism and that $\delta: P(A) \rightarrow \hat{A}$ is an open mapping.

Proposition 2.10. Let $A$ be a Banach-*-algebra with approximate identity. Then $\delta: P(A) \rightarrow \hat{A}$ is open, while $S: \hat{A} \rightarrow\left[C^{*}(A)\right]^{\wedge}$ is a homeomorphism.

Proof. Let $\delta_{\varepsilon}: P\left(C^{*}(A)\right) \rightarrow\left[C^{*}(A)\right]^{\wedge}$ be the canonical mapping corresponding to $\delta . \delta_{\varepsilon}$ is open $[3,3.4]$. We have the following commutative diagram

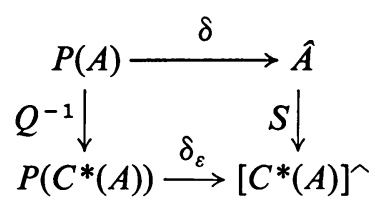

We first indicate the proof of the fact that $S$ is open and continuous, i.e. a homeomorphism. Let $U \subset \hat{A}$ be open. Since $\hat{A}$ has the quotient topology relative to $\delta$, $\delta^{-1}(U)$ is open and hence $Q^{-1}\left(\delta^{-1}(U)\right)$ is open. Since $S(U)=\delta_{\varepsilon}\left(Q^{-1}\left(\delta^{-1}(U)\right)\right), S(U)$ is open, hence $S$ is open. Conversely, if $V \subset\left[C^{*}(A)\right]^{\wedge}$ is open, then $\delta^{-1}\left(S^{-1}(V)\right)$ $=Q\left(\delta_{\varepsilon}^{-1}(V)\right)$ is open. Since $\hat{A}$ has the quotient topology $S^{-1}(V)$ is open. This shows that $S$ is continuous and hence that $S$ is a homeomorphism. From this it follows easily that $\delta$ is open: let $U \subset P(A)$ be open. Since $\delta(U)=S^{-1}\left(\delta_{\varepsilon}\left(Q^{-1}(U)\right)\right.$ the result follows from the above. This completes the proof. 


\section{Tensor products, norms.}

3.1. Let $E_{1}, E_{2}$ be normed linear spaces and $E_{1} \otimes E_{2}$ their algebraic tensor product (see for example [19], or [9]). As is known there is no one canonical way in which norms on $E_{1}, E_{2}$ define a norm on $E_{1} \otimes E_{2}$, although several natural norms on $E_{1} \otimes E_{2}$ can be defined. Among these we mention the least cross norm $\lambda$, defined as follows. Given

$$
t=\sum_{i=1}^{n} x_{i} \otimes y_{i} \in E_{1} \otimes E_{2}, \quad \lambda(t)=\sup \left|\sum_{i=1}^{n} x^{\prime}\left(x_{i}\right) y^{\prime}\left(y_{i}\right)\right|
$$

where the sup is taken over the set of all $x^{\prime}, y^{\prime}$ in the respective unit balls of the dual spaces of $E_{1}, E_{2}$.

Another natural norm on $E_{1} \otimes E_{2}$ is the greatest cross norm $\gamma$, which is defined in the following manner. For $t \in E_{1} \otimes E_{2}, \gamma(t)=\inf \sum\left|x_{i}\right|\left|y_{i}\right|$ with the inf taken over all representatives $\sum_{i=1}^{n} x_{i} \otimes y_{i}=t$. The term 'cross norm' refers to the fact that

$$
\lambda\left(x_{1} \otimes x_{2}\right)=\gamma\left(x_{1} \otimes x_{2}\right)=\left|x_{1}\right|\left|x_{2}\right|
$$

for all $x_{1} \in E_{1}, x_{2} \in E_{2}$.

We say a norm $c$ on $E_{1} \otimes E_{2}$ is ordinary if $\lambda \leqq c \leqq \gamma$. If $c$ is a norm on $E_{1} \otimes E_{2}$ then the normed linear space thus arising will be denoted by $E_{1} \otimes^{c} E_{2}$. The completion of $E_{1} \otimes^{c} E_{2}$ with respect to $c$ is denoted by $E_{1} \otimes_{c} E_{2}$.

3.2. If $E_{1}, E_{2}$ are algebras there is a natural definition of multiplication in $E_{1} \otimes E_{2}$ turning this into an algebra.

If $t_{1}=\sum x_{i} \otimes y_{i}$ and $t_{2}=\sum u_{j} \otimes v_{j}$ are elements in $E_{1} \otimes E_{2}$, then

$$
t_{1} t_{2}=\sum_{i j} x_{i} u_{j} \otimes y_{i} v_{j}
$$

That this is a meaningful concept has been proved by Turumaru [24]. A shorter proof is due to Gil de Lamadrid. We shall reproduce this proof here. It relies on the following

Proposition [1, p. 4]. Let $M: E_{1} \times E_{2} \rightarrow E_{1} \otimes E_{2}$ be the natural mapping $M(x, y)=x \otimes y$. If $G$ is any vector space and $f: E_{1} \times E_{2} \rightarrow G$ is a bilinear mapping, then there is a unique linear mapping $g: E_{1} \otimes E_{2} \rightarrow G$ so that $f=g \circ M$.

With this proposition in mind we can easily prove that the above definition of multiplication is meaningful. Fix $X=\left\{x_{i}\right\}_{i=1}^{n} \subset E_{1}, \quad Y=\left\{y_{i}\right\}_{i=1}^{n} \subset E_{2}$ and define $f_{X Y}: E_{1} \times E_{2} \rightarrow E_{1} \otimes E_{2}$ by

$$
f_{X Y}(x, y)=\sum_{i} x x_{i} \otimes y y_{i}
$$

$f_{X Y}$ is bilinear. Hence there is a unique linear mapping $g_{X Y}: E_{1} \otimes E_{2} \rightarrow E_{1} \otimes E_{2}$ 
with the property that $f_{X Y}(x, y)=g_{X Y}(M(x, y))$ for all $(x, y) \in E_{1} \times E_{2}$, i.e.

$$
\begin{aligned}
g_{X Y}\left(\sum_{j} u_{j} \otimes v_{j}\right) & =g_{X Y}\left(\sum_{j} M\left(u_{j}, v_{j}\right)\right) \\
& =\sum_{j} g_{X Y}\left(M\left(u_{j}, v_{j}\right)\right)=\sum_{j} f_{X Y}\left(u_{j}, v_{j}\right) \\
& =\sum_{j} \sum_{i} u_{j} x_{i} \otimes v_{j} y_{i} \text { for any } \sum_{j} u_{j} \otimes v_{j} \in E_{1} \otimes E_{2} .
\end{aligned}
$$

Repetition of this argument with the fixed factors to the left yields the desired conclusion.

3.3 Whenever we consider $E_{1} \otimes^{c} E_{2}$ or $E_{1} \otimes_{c} E_{2}$, where $E_{1}, E_{2}$ are normed algebras we shall (explicitly or otherwise) assume that $E_{1} \otimes^{c} E_{2}$ is a normed algebra, i.e. that $c$ satisfies

$$
c\left(t_{1} t_{2}\right) \leqq c\left(t_{1}\right) c\left(t_{2}\right) \quad \text { for all } t_{1}, t_{2} \in E_{1} \otimes E_{2} .
$$

We say that such a $c$ is compatible with multiplication on $E_{1} \otimes E_{2}$. The greatest cross norm $\gamma$ is always compatible with multiplication [4, Theorem 1] while there are examples of algebras on which $\lambda$ is not compatible with multiplication $[7, \S 5]$. Under certain circumstances, however, $\lambda$ does yield a Banach algebra [8], [22].

3.4. If $E_{1}, E_{2}$ are $*$-algebras, then an involution can be defined on $E_{1} \otimes E_{2}$ in this fashion:

$$
\text { if } t=\sum x_{i} \otimes y_{i} \in E_{1} \otimes E_{2} \text {, then } t^{*}=\sum x_{i}^{*} \otimes y_{i}^{*} .
$$

A method similar to that employed in 3.2 will show that $*$ as defined here is a welldefined involution. A norm $c$ on $E_{1} \otimes E_{2}$ is compatible with involution on $E_{1} \otimes E_{2}$ if $c(t)=c\left(t^{*}\right)$ for all $t \in E_{1} \otimes E_{2}$. Both $\lambda$ and $\gamma$ are compatible with involution.

3.5. If $E_{1}, E_{2}$ are $C^{*}$-algebras then there are several norms $c$ that turn $E_{1} \otimes_{c} E_{2}$ into a $C^{*}$-algebra. We shall pay special attention to two such norms.

The least $C^{*}$-norm $\alpha$. Let $E_{1}, E_{2}$ be $C^{*}$-algebras and

$$
\mu_{1}: E_{1} \rightarrow L\left(H_{1}\right), \quad \mu_{2}: E_{2} \rightarrow L\left(H_{2}\right)
$$

be representations. If $t=\sum x_{i} \otimes y_{i} \in E_{1} \otimes E_{2}$, then we define

$$
\mu_{1} \otimes \mu_{2}(t)=\sum \mu_{1}\left(x_{i}\right) \otimes \mu_{2}\left(y_{i}\right) \in L\left(H_{1}\right) \otimes L\left(H_{2}\right) \subset L\left(H_{1} \otimes_{\sigma} H_{2}\right)
$$

where $\sigma$ is the canonical norm induced by the product of the inner products on $H_{1}$ and $H_{2} . \alpha$ is defined as follows:

$$
\alpha(t)=\left|\mu_{1} \otimes \mu_{2}(t)\right| \quad \text { for any } t \in E_{1} \otimes E_{2}
$$

where $\mu_{1}, \mu_{2}$ are faithful representations. Wulfsohn [25] shows that this definition is independent of the choice of representations.

The greatest $C^{*}$-norm $v$. This norm was introduced by Guichardet [11] and defined by $\nu(t)=\sup _{\pi}|\pi(t)|$ where $\pi$ ranges over the set of all representations of $E_{1} \otimes E_{2}$ satisfying the condition

$$
\left|\pi\left(x_{1} \otimes x_{2}\right)\right| \leqq\left|x_{1}\right|\left|x_{2}\right| \text { for all } x_{i} \in E_{i}(i=1,2) .
$$


Clearly $\alpha \leqq \nu$. The question of equality of the two norms has been studied by Takesaki [23]. He says that a $C^{*}$-algebra $A$ has property (T) if $A \otimes_{\alpha} B=A \otimes_{\nu} B$ for any $C^{*}$-algebra $B$ and shows that the class of $C^{*}$-algebras with property $(T)$ properly contains the class of $C^{*}$-algebras of type $\mathrm{I}$.

We remark that both $\alpha$ and $\nu$ are cross norms. From the definition of $\nu$ and the fact that $\alpha \leqq \nu$ it follows that it suffices to show this for $\alpha$; and for $\alpha$ this is a consequence of [8, Theorem 1.2].

\section{The enveloping algebra of a tensor product.}

4.1. In this section we show that in a great many cases the enveloping algebra of a tensor product can be identified with a tensor product of the respective enveloping algebras of the Banach-*-algebras in question.

But first we state and prove a result concerning extension of certain representations. This result will be helpful later.

4.2. As we indicated in 3.5 , if $E_{1}, E_{2}$ are Banach-*-algebras and $\mu_{i}: E_{i} \rightarrow L\left(H_{\mu_{i}}\right)$ representations, then we can define

$$
\mu_{1} \otimes \mu_{2}: E_{1} \otimes E_{2} \rightarrow L\left(H_{\mu_{1}} \otimes_{\sigma} H_{\mu_{2}}\right)
$$

by

$$
\mu_{1} \otimes \mu_{2}\left(\sum x_{i} \otimes y_{i}\right)=\sum \mu_{1}\left(x_{i}\right) \otimes \mu_{2}\left(y_{i}\right) \in L\left(H_{\mu_{1}} \otimes_{\sigma} H_{\mu_{2}}\right)
$$

for any $\sum x_{i} \otimes y_{i} \in E_{1} \otimes E_{2}$. If $c$ is a norm on $E_{1} \otimes E_{2}$ then $\mu_{1} \otimes \mu_{2}$ can be extended to all of $E_{1} \otimes_{c} E_{2}$ under rather mild restrictions on $c$. We have the following

Proposition 4.3. Let $E_{1}, E_{2}$ be Banach-*-algebras with approximate identities and $c$ be an ordinary norm on $E_{1} \otimes E_{2}$, compatible with multiplication and involution. If $\mu_{i}$ is a representation of $E_{i}(i=1,2)$ then $\mu_{1} \otimes \mu_{2}$ can be uniquely extended to $E_{1} \otimes_{c} E_{2}$. The extended representation will also be denoted by $\mu_{1} \otimes \mu_{2}$.

The proof of this proposition requires the following

Lemma 4.4. Let $E_{1}, E_{2}$ and $c$ be as in Proposition 4.3 and let $p_{i}$ be a continuous positive linear functional on $E_{i}(i=1,2) . p_{1} \otimes p_{2}$ defined on $E_{1} \otimes E_{2}$ by

$$
p_{1} \otimes p_{2}\left(\sum x_{i} \otimes y_{i}\right)=\sum p_{1}\left(x_{i}\right) p_{2}\left(y_{i}\right)
$$

for any $\sum x_{i} \otimes y_{i} \in E_{1} \otimes E_{2}$ can be uniquely extended to a positive continuous linear functional on $E_{1} \otimes_{c} E_{2}$ which will also be denoted by $p_{1} \otimes p_{2}$.

The proof of this lemma is a straightforward adaptation to the present situation of the proof of [24, Lemma 1].

Proof of Proposition 4.3. Given any representation $\pi$ of an algebra $A$ there are representations $\pi_{0}$ and $\pi_{i}$ such that $\pi=\pi_{0} \oplus\left(\sum \oplus \pi_{i}\right)$ where $\pi_{0}$ is trivial and $\pi_{i}$ is 
cyclic [3, 2.2.6 and 2.2.7]. Using this decomposition we get that

$$
\begin{aligned}
\mu_{1} \otimes \mu_{2} & =\left(\mu_{0}^{1} \oplus\left(\sum \oplus \mu_{i}^{1}\right)\right) \otimes\left(\mu_{0}^{2} \oplus\left(\sum \oplus \mu_{j}^{2}\right)\right) \\
& =\sum_{i j} \oplus\left(\mu_{i}^{1} \otimes \mu_{j}^{2}\right)
\end{aligned}
$$

where each $\mu_{i}^{1}, \mu_{j}^{2}$ is either cyclic or trivial. Since it suffices to show that each $\mu_{i}^{1} \otimes \mu_{j}^{2}$ can be extended we can assume without loss of generality that $\mu_{1}$ and $\mu_{2}$ are cyclic with cyclic vectors $a_{1} \in H_{\mu_{1}}$ and $a_{2} \in H_{\mu_{2}}$, respectively (obviously the trivial representation can be extended). Define

$$
p_{i}\left(x_{i}\right)=\left(\mu_{i}\left(x_{i}\right) a_{i} \mid a_{i}\right) \text { for all } x_{i} \in E_{i}(i=1,2) .
$$

By Lemma $4.4 p_{1} \otimes p_{2}$ defines a positive continuous linear functional on $E_{1} \otimes_{c} E_{2}$. Let $\pi^{\prime}$ be the representation in a Hilbert space $H$ generated by $p_{1} \otimes p_{2}$ and let $d$ be a cyclic vector for $\pi^{\prime}$ such that

$$
p_{1} \otimes p_{2}(t)=\left(\pi^{\prime}(t) d \mid d\right) \quad \text { for all } t \in E_{1} \otimes_{c} E_{2}
$$

(see [3, Proposition 2.4.4]).

Since $E_{1} \otimes^{c} E_{2}$ is dense in $E_{1} \otimes_{c} E_{2}$ and $d$ is cyclic

$$
H_{0}=\left\{\pi^{\prime}(s) d ; s \in E_{1} \otimes^{c} E_{2}\right\}
$$

is dense in $H$.

We shall define an isometry onto, $U: H \rightarrow H_{\mu_{1}} \otimes_{\sigma} H_{\mu_{2}}$. If $h \in H_{0}$, then $h=\pi^{\prime}(s) d$ for some $s \in E_{1} \otimes^{c} E_{2}$; we let

$$
U h=\mu_{1} \otimes \mu_{2}(s)\left(a_{1} \otimes a_{2}\right) .
$$

An easy computation shows that

$$
\begin{aligned}
|h|^{2} & =\left(\pi^{\prime}(s) d \mid \pi^{\prime}(s) d\right)=\left(\pi^{\prime}\left(s^{*} s\right) d \mid d\right) \\
& =\left(\mu_{1} \otimes \mu_{2}\left(s^{*} s\right)\left(a_{1} \otimes a_{2}\right) \mid\left(a_{1} \otimes a_{2}\right)\right) \\
& =|U h|^{2}
\end{aligned}
$$

so that $U$ is an isometry. To conclude that $U$ can be extended to a surjective mapping it suffices to observe that

$$
H_{1}=\left\{\mu_{1} \otimes \mu_{2}(s)\left(a_{1} \otimes a_{2}\right) ; s \in E_{1} \otimes^{c} E_{2}\right\}
$$

is dense in $H_{\mu_{1}} \otimes_{\sigma} H_{\mu_{2}}$, since $a_{i}$ is cyclic for $\mu_{i}$ in $H_{\mu_{i}}(i=1,2)$.

We denote the extended isometry also by $U$ and define

by

$$
\pi: E_{1} \otimes_{c} E_{2} \rightarrow L\left(H_{\mu_{1}} \otimes_{\sigma} H_{\mu_{2}}\right)
$$

$$
\pi(t) U=U \pi^{\prime}(t) \text { for all } t \in E_{1} \otimes_{c} E_{2} .
$$

We claim that $\pi$ is an extension of $\mu_{1} \otimes \mu_{2}$; to show this let $s \in E_{1} \otimes^{c} E_{2}$ and $h \in H_{0}$, i.e. $h=\pi^{\prime}\left(s_{1}\right) d$ for some $s_{1} \in E_{1} \otimes^{c} E_{2}$. Then

$$
\begin{aligned}
\mu_{1} \otimes \mu_{2}(s) U h & =\mu_{1} \otimes \mu_{2}(s) \mu_{1} \otimes \mu_{2}\left(s_{1}\right)\left(a_{1} \otimes a_{2}\right) \\
& =\mu_{1} \otimes \mu_{2}\left(s s_{1}\right)\left(a_{1} \otimes a_{2}\right)=U\left[\pi^{\prime}\left(s s_{1}\right) d\right] \\
& =U \pi^{\prime}(s) \pi^{\prime}\left(s_{1}\right) d=U \pi^{\prime}(s) h=\pi(s) U h
\end{aligned}
$$


for all $s \in E_{1} \otimes^{c} E_{2}$ and $h \in H_{0}$. But $U H_{0}=H$, which is dense in $H_{\mu_{1}} \otimes_{\sigma} H_{\mu_{2}}$. It follows that $\mu_{1} \otimes \mu_{2}$ and $\pi$ coincide wherever both are defined; consequently $\pi$ extends $\mu_{1} \otimes \mu_{2}$. This completes the proof.

Proposition 4.3 will be used to derive some corollaries from the following main theorem. Before stating and proving this theorem we need the following definitions and lemmas.

4.5. Suppose $E_{1}, E_{2}$ are Banach-*-algebras with approximate identities and suppose $c$ is a cross norm on $E_{1} \otimes E_{2}$ which is compatible with multiplication and involution (see 3.3 and 3.4). Suppose further that $C^{*}\left(E_{i}\right)$ denote the enveloping algebras and $\varepsilon_{i}$ the enveloping mappings (see 2.8). Let

$$
\varphi: E_{1} \otimes E_{2} \rightarrow C^{*}\left(E_{1}\right) \otimes_{\nu} C^{*}\left(E_{2}\right)
$$

be the natural mapping, i.e.

$$
\varphi\left(\sum x_{i} \otimes y_{i}\right)=\sum \varepsilon_{1}\left(x_{i}\right) \otimes \varepsilon_{2}\left(y_{i}\right)
$$

for any $\sum x_{i} \otimes y_{i} \in E_{1} \otimes E_{2}$. We require $\varphi$ to be extendible to all of $E_{1} \otimes_{c} E_{2}$. Since $C^{*}\left(E_{1}\right) \otimes_{\nu} C^{*}\left(E_{i}\right)$ is a $C^{*}$-algebra a necessary and sufficient condition for this to be possible is

$$
\nu(\varphi(t)) \leqq c(t) \text { for all } t \in E_{1} \otimes^{c} E_{2}
$$

[3, p. 7].

LEMMA 4.7. Let $E_{1}, E_{2}$ be Banach-*-algebras with approximate identities and c a cross norm on $E_{1} \otimes E_{2}$ compatible with multiplication and involution. Let $\pi$ be a representation of $E_{1} \otimes_{c} E_{2}$ in a Hilbert space $H$. With $\pi$ we can associate representations $\pi_{i}$ of $E_{i}(i=1,2)$ in $H$ with the properties

(i) $\pi\left(u_{a} \otimes y\right) \rightarrow \pi_{2}(y)$ strongly for any $y \in E_{2}$ and any approximate identity $\left\{u_{a}\right\} \subset E_{1}$.

(ii) $\pi\left(x \otimes v_{b}\right) \rightarrow \pi_{1}(x)$ strongly for any $x \in E_{1}$ and any approximate identity $\left\{v_{b}\right\} \subset E_{2}$.

(iii) $\pi\left(x_{1} \otimes x_{2}\right)=\pi_{1}\left(x_{1}\right) \pi_{2}\left(x_{2}\right)=\pi_{2}\left(x_{2}\right) \pi_{1}\left(x_{1}\right)$ for all $x_{i} \in E_{i}$.

The proof of Lemma 4.7 is identical with that of Proposition 1 of [10] and will therefore be omitted.

$\pi_{i}$ is called the restriction of $\pi$ to $E_{i}$. We can now prove the main result of this section.

THEOREM 4.8. If $E_{1}, E_{2}$ are Banach-*-algebras with approximate identities and $c$ a cross norm on $E_{1} \otimes E_{2}$ which is compatible with multiplication and involution and which satisfies condition (4.6), then

$$
C^{*}\left(E_{1} \otimes_{c} E_{2}\right)=C^{*}\left(E_{1}\right) \otimes_{\nu} C^{*}\left(E_{2}\right)
$$

where $=$ indicates the existence of a surjective isometric isomorphism. 
Proof. We first show that $\nu(\varphi(s))=|\varepsilon(s)|$ for any $s \in E_{1} \otimes^{c} E_{2}$ where $|\cdot|$ is the norm in $C^{*}\left(E_{1} \otimes_{c} E_{2}\right) .|\varepsilon(s)| \leqq \nu(\varphi(s))$ : Let $\pi$ be a faithful representation of $C^{*}\left(E_{1} \otimes_{c} E_{2}\right)$ and let $\rho$ be the corresponding representation of $E_{1} \otimes_{c} E_{2}$, i.e. $\rho=\pi \circ \varepsilon$, where $\varepsilon$ is the enveloping mapping. Let $\rho_{i}$ be the restriction of $\rho$ to $E_{i}$, as defined in Lemma 4.7 and let $\pi_{i}$ be the representation of $C^{*}\left(E_{i}\right)$ corresponding to $\rho_{i}$, i.e. $\rho_{i}=\pi_{i} \circ \varepsilon_{i}$, where $\varepsilon_{i}$ is the enveloping mapping of $E_{i}, i=1,2$. For

$$
t=\sum x_{j} \otimes y_{j} \in C^{*}\left(E_{1}\right) \otimes C^{*}\left(E_{2}\right)
$$

define

$$
\tau(t)=\sum \pi_{1}\left(x_{j}\right) \pi_{2}\left(y_{j}\right)
$$

Since $\pi_{1}$ and $\pi_{2}$ are commuting representations (Lemma 4.7(iii)) $\tau$ is a representation of $C^{*}\left(E_{1}\right) \otimes C^{*}\left(E_{2}\right)$. Moreover, $\tau \circ \varphi$ is the restriction of $\rho$ to $E_{1} \otimes E_{2}$ : if $\sum x_{i} \otimes y_{i} \in E_{1} \otimes E_{2}$, then

$$
\begin{aligned}
\rho\left(\sum x_{i} \otimes y_{i}\right) & =\sum \rho\left(x_{i} \otimes y_{i}\right)=\sum \rho_{1}\left(x_{i}\right) \rho_{2}\left(y_{i}\right)=\sum\left(\pi_{1} \circ \varepsilon_{1}\left(x_{i}\right)\right)\left(\pi_{2} \circ \varepsilon_{2}\left(y_{i}\right)\right) \\
& =\tau\left(\sum \varepsilon_{1}\left(x_{i}\right) \otimes \varepsilon_{2}\left(y_{i}\right)\right)=\tau \circ \varphi\left(\sum x_{i} \otimes y_{i}\right) .
\end{aligned}
$$

Since

$$
\left|\tau\left(x_{1} \otimes x_{2}\right)\right|=\left|\pi_{1}\left(x_{1}\right) \pi_{2}\left(x_{2}\right)\right| \leqq\left|\pi_{1}\left(x_{1}\right)\right|\left|\pi_{2}\left(x_{2}\right)\right| \leqq\left|x_{1}\right|\left|x_{2}\right|
$$

for all $x_{i} \in E_{i},|\tau(t)| \leqq \nu(t)$ for any $t \in C^{*}\left(E_{1}\right) \otimes C^{*}\left(E_{2}\right)$. Consequently, if $s \in E_{1} \otimes{ }^{c} E_{2}$, then

$$
|\varepsilon(s)|=|\pi(\varepsilon(s))|=|\rho(s)|=|\tau \circ \varphi(s)| \leqq \nu(\varphi(s)) .
$$

$\nu(\varphi(s)) \leqq|\varepsilon(s)|$ : To get this inequality we use condition (4.6). Let $\pi$ be a faithful representation of $C^{*}\left(E_{1}\right) \otimes_{\nu} C^{*}\left(E_{2}\right)$. Then $\rho=\pi \circ \varphi$ is a representation of $E_{1} \otimes_{c} E_{2}$. Let $\mu$ be the representation of $C^{*}\left(E_{1} \otimes_{c} E_{2}\right)$ corresponding to $\rho$; if $s \in E_{1} \otimes_{c} E_{2}$, then

$$
\nu(\varphi(s))=|\pi(\varphi(s))|=|\rho(s)|=|\mu \circ \varepsilon(s)| \leqq|\varepsilon(s)| .
$$

We have shown that

$$
|\varepsilon(s)|=\nu(\varphi(s)) \text { for all } s \in E_{1} \otimes^{c} E_{2} .
$$

Since $\varepsilon\left(E_{1} \otimes^{c} E_{2}\right)$ is dense in $C^{*}\left(E_{1} \otimes_{c} E_{2}\right)$ and $\varphi\left(E_{1} \otimes^{c} E_{2}\right)$ is dense in $C^{*}(E) \otimes_{\nu} C^{*}(F)$ it follows that

$$
|\varepsilon(s)|=\nu(\varphi(s)) \text { for all } s \in E_{1} \otimes_{c} E_{2} .
$$

Moreover, $\varepsilon \circ \varphi^{-1}$ is seen to define an isometric isomorphism of $C^{*}\left(E_{1}\right) \otimes_{\nu} C^{*}\left(E_{2}\right)$ onto $C^{*}\left(E_{1} \otimes_{c} E_{2}\right)$. This completes the proof.

Condition (4.6) is somewhat artificial looking. However, if we restrict the algebra under consideration, then this condition is satisfied by a natural class of norms on $E_{1} \otimes E_{2}$. We have the following 
COROLlARY 4.9 [26]. If $E_{1}$ or $E_{2}$ is of type I and c is an ordinary norm compatible with multiplication and involution, then

$$
C^{*}\left(E_{1} \otimes_{c} E_{2}\right)=C^{*}\left(E_{1}\right) \otimes_{\alpha} C^{*}\left(E_{2}\right) .
$$

Proof. Takesaki [23] has shown that if $E_{1}$ or $E_{2}$ is of type I then $\alpha=\nu$ (see 3.5). From the definition of $\alpha$ and Proposition 4.3 it follows that $\varepsilon_{1} \otimes \varepsilon_{2} \equiv \varphi$ can be extended to all of $E_{1} \otimes_{c} E_{2}$, i.e. $c$ satisfies condition (4.6). The result then follows from the above theorem. then

We also note that condition (4.6) is satisfied by $\gamma$ : if $t=\sum x_{i} \otimes y_{i} \in E_{1} \otimes^{\gamma} E_{2}$,

$$
\begin{aligned}
\nu(\varphi(t)) & =\nu\left(\sum \varepsilon_{1}\left(x_{i}\right) \otimes \varepsilon_{2}\left(y_{i}\right)\right) \\
& \leqq \sum \nu\left(\varepsilon_{1}\left(x_{i}\right) \otimes \varepsilon_{2}\left(y_{i}\right)\right) \\
& \leqq \sum\left|\varepsilon_{1}\left(x_{i}\right)\right|\left|\varepsilon_{2}\left(y_{i}\right)\right| \leqq \sum\left|x_{i}\right|\left|y_{i}\right|
\end{aligned}
$$

This being true for any representative $\sum x_{i} \otimes y_{i}$ of $t$ we get

$$
\nu(\varphi(t)) \leqq \inf \sum\left|x_{i}\right|\left|y_{i}\right|=\gamma(t)
$$

for all $t \in E_{1} \otimes E_{2}$.

It follows that Theorem 3 [16] is also a corollary of Theorem 4.8.

\section{Spectra of tensor products.}

5.1. In this section we study the relationships between $\hat{E}_{1}, \hat{E}_{2}$ and $\left(E_{1} \otimes_{c} E_{2}\right)^{\wedge}$. Proposition 4.3 suggests that $\hat{E}_{1} \times \hat{E}_{2} \subset\left(E_{1} \otimes_{c} E_{2}\right)^{\wedge}$ in a natural fashion. We show that this is the case and investigate the problem of equality of $\hat{E}_{1} \times \hat{E}_{2}$ and $\left(E_{1} \otimes_{c} E_{2}\right)^{\wedge}$.

5.2. Our first result concerns the embedding of $\hat{E}_{1} \times \hat{E}_{2}$ into $\left(E_{1} \otimes_{c} E_{2}\right)^{\wedge}$.

Recall that if $E$ is a Banach-*-algebra with approximate identity then $\hat{E}$ and $\left[C^{*}(E)\right]^{\wedge}$ are homeomorphic (Proposition 2.10). This fact will be of help to us in the following.

We shall use the fullowing notation: if $S_{i}, V_{i}(i=1,2)$ are sets and $f_{i}: S_{i} \rightarrow V_{i}$ are functions then $f_{1} \times f_{2}: S_{1} \times S_{2} \rightarrow V_{1} \times V_{2}$ is defined by

$$
f_{1} \times f_{2}\left(s_{1}, s_{2}\right)=\left(f_{1}\left(s_{1}\right), f_{2}\left(s_{2}\right)\right) \text { for all }\left(s_{1}, s_{2}\right) \in S_{1} \times S_{2} .
$$

We also note the following fact the proof of which is straightforward.

Lemma 5.3. Let $S_{i}, V_{i}(i=1,2)$ be topological spaces and $f_{i}: S_{i} \rightarrow V_{i}$ be open mappings. Then $f_{1} \times f_{2}: S_{1} \times S_{2} \rightarrow V_{1} \times V_{2}$ is open.

Proposition 5.4. Let $E_{1}, E_{2}$ be Banach-*-algebras with approximate identities and $c$ an ordinary norm compatible with multiplication and involution on $E_{1} \otimes E_{2}$. For every $\bar{\mu}_{1} \in \hat{E}_{1}, \bar{\mu}_{2} \in \hat{E}_{2}$ define $G$ by $G\left(\bar{\mu}_{1}, \bar{\mu}_{2}\right)=\left(\mu_{1} \otimes \mu_{2}\right)^{\wedge}$. Then

(i) $G\left(\hat{E}_{1} \times \hat{E}_{2}\right) \subset\left(E_{1} \otimes_{c} E_{2}\right)^{\wedge}$. 
(ii) $G$ is $1-1$.

(iii) $G$ is continuous.

(iv) if c also satisfies condition (4.6), then $G^{-1}$ is continuous.

Proof. We first note that $G$ is well defined; in fact if $\mu_{1}^{\prime} \cong \mu_{1}^{\prime \prime}$ and $\mu_{2}^{\prime} \cong \mu_{2}^{\prime \prime}$, then $\mu_{1}^{\prime} \otimes \mu_{2}^{\prime} \cong \mu_{1}^{\prime \prime} \otimes \mu_{2}^{\prime \prime}:$ suppose

$$
\mu_{i}^{\prime}\left(x_{i}\right) U_{i} h_{i}=U_{i} \mu_{i}^{\prime \prime}\left(x_{i}\right) h_{i}
$$

for all $h_{i} \in H_{\mu_{i}^{*}}$ and all $x_{i} \in E_{i}(i=1,2)$ where $U_{i}: H_{\mu_{i}^{*}} \rightarrow H_{\mu_{i}^{*}}(i=1,2)$ is an isometry onto. $U_{1} \otimes U_{2}$ defines an isometry of $H_{\mu_{1}^{\prime \prime}} \otimes_{\sigma} H_{\mu_{2}^{\prime \prime}}$ onto $H_{\mu_{1}^{\prime}} \otimes_{\sigma} H_{\mu_{2}^{\prime}}$. A straightforward algebraic manipulation and a simple continuity argument then show that

$$
\mu_{1}^{\prime} \otimes \mu_{2}^{\prime}(t)\left(U_{1} \otimes U_{2}\right)(h)=U_{1} \otimes U_{2}\left(\mu_{1}^{\prime \prime} \otimes \mu_{2}^{\prime \prime}\right)(t) h
$$

for every $t \in E_{1} \otimes_{c} E_{2}$ and every $h \in H_{\mu_{1}^{\prime \prime}} \otimes_{\sigma} H_{\mu_{2}^{\prime \prime}}$.

If $\mu_{i} \in \bar{\mu}_{i} \in \hat{E}_{i}(i=1,2)$, then $w^{*}\left[\mu_{i}\left(E_{i}\right)\right]=L\left(H_{\mu_{i}}\right)$ (Proposition 2.3) so that

$$
\begin{aligned}
w^{*}\left[\mu_{1} \otimes \mu_{2}\left(E_{1} \otimes_{c} E_{2}\right)\right] & =w^{*}\left[L\left(H_{\mu_{1}}\right) \otimes L\left(H_{\mu_{2}}\right)\right] \\
& =L\left(H_{\mu_{1}} \otimes_{\sigma} H_{\mu_{2}}\right) \quad[2, \text { p. 28] }
\end{aligned}
$$

This shows that $\left(\mu_{1} \otimes \mu_{2}\right)^{\wedge} \in\left(E_{1} \otimes_{c} E_{2}\right)^{\wedge}$.

To prove (ii) let $\bar{\mu}_{i}^{\prime}, \bar{\mu}_{i}^{\prime \prime} \in \hat{E}_{i}$ be chosen so that $\left(\mu_{1}^{\prime} \otimes \mu_{2}^{\prime}\right)^{\wedge}=\left(\mu_{1}^{\prime \prime} \otimes \mu_{2}^{\prime \prime}\right)^{\wedge} \in\left(E_{1} \otimes_{c} E_{2}\right)^{\wedge}$. We first observe that if $\pi_{i}^{(j)}$ is the restriction of $\mu_{1}^{(j)} \otimes \mu_{2}^{(j)}$ to $E_{i}(i, j=1,2)$, then $\pi_{i}^{(j)}$ is a multiple of $\mu_{i}^{(j)}$. This is true since $\pi_{1}^{(j)}=\mu_{1}^{(j)} \otimes I_{1}^{(j)}$ where $I_{1}^{(j)}$ is the identity on $H_{\mu_{2}^{(j)}}$ and since a similar relationship holds between $\pi_{2}^{(j)}$ and $\mu_{2}^{(j)}$. If $\mu_{1}^{\prime} \otimes \mu_{2}^{\prime}$ $\cong \mu_{1}^{\prime \prime} \otimes \mu_{2}^{\prime \prime}$ then $\pi_{i}^{\prime} \cong \pi_{i}^{\prime \prime}$ : suppose that for some isometry $U$ we have

$$
U \mu_{1}^{\prime} \otimes \mu_{2}^{\prime}(t) h=\mu_{1}^{\prime \prime} \otimes \mu_{2}^{\prime \prime}(t) U h
$$

for all $t \in E_{1} \otimes_{c} E_{2}$ and $h \in H_{\mu_{1}^{\prime}} \otimes \mu_{2}^{\prime}$. Then for all $x_{1} \in E_{1}$ and $h \in H_{\mu_{1}^{\prime} \otimes \mu_{2}^{\prime}}$ we have

$$
\begin{aligned}
U \pi_{1}^{\prime}\left(x_{1}\right) h & =U \lim _{\beta}\left(\mu_{1}^{\prime}\left(x_{1}\right) \otimes \mu_{2}^{\prime}\left(v_{\beta}\right)\right) h \\
& =\lim _{\beta}\left(\mu_{1}^{\prime \prime} \otimes \mu_{2}^{\prime \prime}\left(x_{1} \otimes v_{\beta}\right) U h\right) \\
& =\lim _{\beta}\left(\mu_{1}^{\prime \prime}\left(x_{1}\right) \otimes \mu_{2}^{\prime \prime}\left(v_{\beta}\right)(U h)\right) \\
& =\pi_{1}^{\prime \prime}\left(x_{1}\right) U h
\end{aligned}
$$

where $\left\{v_{\beta}\right\}$ is an approximate identity for $E_{2}$. This shows $\pi_{1}^{\prime} \cong \pi_{1}^{\prime \prime}$. Similarly $\pi_{2}^{\prime} \cong \pi_{2}^{\prime \prime}$. The equivalence of $\pi_{i}^{\prime}$ and $\pi_{i}^{\prime \prime}$ implies the quasi-equivalence of $\mu_{i}^{\prime}$ and $\mu_{i}^{\prime \prime}[3,5.3]$. But two topologically irreducible quasi-equivalent representations are equivalent [3, 5.3.3]. This proves (ii).

To prove (iii) let $\delta_{i}: P\left(E_{i}\right) \rightarrow \hat{E}_{i}$ be the open continuous canonical mappings (see Proposition 2.10) and let $f \equiv \delta_{1} \times \delta_{2}$. Then $f$ is open and continuous (Lemma 5.3). If we define $g: P\left(E_{1}\right) \times P\left(E_{2}\right) \rightarrow P\left(E_{1} \otimes_{c} E_{2}\right)$ by $g\left(p_{1}, p_{2}\right)=p_{1} \otimes p_{2}$ (this 
mapping was considered in Lemma 4.4) then we have the following commutative diagram

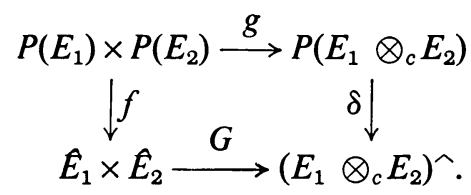

Since $f$ is open and continuous $G$ is continuous iff $G \circ f$ is continuous [13, Theorems 8,9, p. 95]. Moreover, $G \circ f=\delta \circ g$ and $\delta$ open and continuous imply that it suffices to show that $g$ is continuous. But this is a simple consequence of the facts that pure states are uniformly bounded and $E_{1} \otimes^{c} E_{2}$ is dense in $E_{1} \otimes_{c} E_{2}$.

In the proof of (iv) we shall make use of Theorem 4.8. With the additional assumption on $c$ we know that $C^{*}\left(E_{1} \otimes_{c} E_{2}\right)=C^{*}\left(E_{1}\right) \otimes_{\nu} C^{*}\left(E_{2}\right)$. Since

$$
\left[C^{*}\left(E_{1} \otimes_{c} E_{2}\right)\right]^{\wedge}=\left(E_{1} \otimes_{c} E_{2}\right)^{\wedge} \quad \text { and } \quad E_{i}=\left[C^{*}\left(E_{i}\right)\right]^{\wedge} \quad(i=1,2)
$$

(= indicates existence of a homeomorphism) it is clear that it suffices to show that the mapping $\mu \otimes \nu \rightarrow \mu$ from (a subset of) $\left[C^{*}\left(E_{1} \otimes_{c} E_{2}\right)\right]^{\wedge}$ into $\left[C^{*}\left(E_{1}\right)\right]^{\wedge}$ is continuous. And this can be done by means of a slight variation of an argument due to Wulfsohn [25, Lemma 16].

Every open set in the spectrum of a $C^{*}$-algebra $A$ is of the form

$$
\hat{A}^{I}=\left\{\bar{\pi} \in \hat{A} ;\left.\pi\right|_{I} \not \equiv 0\right\}
$$

where $I$ is a closed 2-sided ideal [3, p. 62]. Let $G_{1}:\left[C^{*}\left(E_{1}\right)\right]^{\wedge} \times\left[C^{*}\left(E_{2}\right)\right]^{\wedge} \rightarrow$ $\left[C^{*}\left(E_{1} \otimes_{c} E_{2}\right)\right]^{\wedge}$ denote the mapping corresponding to $G$ (see Proposition 4.3) and $h_{i}:\left[C^{*}\left(E_{1}\right)\right]^{\wedge} \times\left[C^{*}\left(E_{2}\right)\right]^{\wedge} \rightarrow\left[C^{*}\left(E_{i}\right)\right]^{\wedge}$ the projection. Let $\left[C^{*}\left(E_{1}\right)\right]^{\wedge I}$ be an open set in $\left[C^{*}\left(E_{1}\right)\right]^{\wedge}$. Its pre-image in $\left[C^{*}\left(E_{1} \otimes_{c} E_{2}\right)\right]^{\wedge}$ is $G_{1}\left(h_{1}^{-1}\left[C^{*}\left(E_{1}\right)\right]^{\wedge I}\right)$.

Note that $\left(I \otimes C^{*}\left(E_{2}\right), \nu\right)$, the $\nu$-closure of the algebraic tensor product $I \otimes C^{*}\left(E_{2}\right)$ is a closed 2-sided ideal in $C^{*}\left(E_{1} \otimes_{c} E_{2}\right)$. By the above, to show that $G_{1}^{-1}$ is continuous it suffices to identify $G_{1}\left(h_{1}^{-1}\left[C^{*}\left(E_{1}\right)\right]^{\wedge I}\right)$ with

$$
\left[C^{*}\left(E_{1} \otimes_{c} E_{2}\right)\right]^{\wedge\left(I \otimes C^{*}\left(E_{2}\right), v\right)} \cap G_{1}\left(\left[C^{*}\left(E_{1}\right)\right]^{\wedge} \times\left[C^{*}\left(E_{2}\right)\right]^{\wedge}\right) .
$$

If $(\mu \otimes \nu)^{\wedge} \in G_{1}\left(h_{1}^{-1}\left[C^{*}\left(E_{1}\right)\right]^{\wedge I}\right)$, then $\mu \in \bar{\mu}$ does not vanish on $I$ and consequently $\mu \otimes \nu$ does not vanish on $I \otimes C^{*}\left(E_{2}\right)$ so

$$
(\mu \otimes \nu)^{\wedge} \in\left[C^{*}\left(E_{1} \otimes_{c} E_{2}\right)\right]^{\wedge\left(I \otimes C^{*}\left(E_{2}\right), v\right)} \cap G_{1}\left(\left[C^{*}\left(E_{1}\right)\right]^{\wedge} \times\left[C^{*}\left(E_{2}\right)\right]^{\wedge}\right) .
$$

Conversely, if $(\mu \otimes \nu)^{\wedge}$ belongs to this last mentioned set we must show $\bar{\mu} \in\left[C^{*}\left(E_{1}\right)\right]^{\wedge I}$. Since if $\mu \in \bar{\mu}$ and $\nu \in \bar{\nu},\left.\mu \otimes \nu\right|_{\left(I \otimes C^{*}\left(E_{2}\right), v\right)}=\left.\mu\right|_{I} \otimes \nu$ where the representation on the right-hand side is taken in the sense of Proposition 4.3 and since $\left.\mu \otimes \nu\right|_{\left(I \otimes C^{*}\left(E_{2}\right), v\right)} \not \equiv 0$ it follows that $\left.\mu\right|_{I} \not \equiv 0$. This completes the proof.

Because of this proposition it is natural to investigate when $G$ is an homeomorphism, i.e. is onto. As it turns out, a sufficient condition is that $E_{1}$ or $E_{2}$ be of type $\mathrm{I}$. This condition is also necessary if $E_{1}$ and $E_{2}$ are separable. These facts can 
be proved directly. However, we shall make some shortcuts by appealing to existing results via the enveloping algebras.

THEOREM 5.5. If $E_{1}, E_{2}$ are Banach-*-algebras with approximate identities and one of them is of type $\mathrm{I}$ and if $c$ is an ordinary norm on $E_{1} \otimes E_{2}$ compatible with multiplication and involution, then

$$
\hat{E}_{1} \times \hat{E}_{2}=\left(E_{1} \otimes_{c} E_{2}\right)^{\wedge},
$$

i.e. $G$ as defined in Proposition 5.4 is a homeomorphism.

Proof. $C^{*}\left(E_{1} \otimes_{c} E_{2}\right)=C^{*}\left(E_{1}\right) \otimes_{\alpha} C^{*}\left(E_{2}\right)$ (Corollary 4.9). The conclusion then follows from [25, Lemma 13] and Proposition 2.10 (since $E_{1}$, say, is of type I iff $C^{*}\left(E_{1}\right)$ is of type I).

As a partial converse we have the following

THEOREM 5.6. Let $E_{1}, E_{2}$ be separable Banach-*-algebras with approximate identities and $c$ a cross norm on $E_{1} \otimes E_{2}$ compatible with multiplication and involution and satisfying condition (4.6). If $G: \hat{E}_{1} \times \hat{E}_{2} \rightarrow\left(E_{1} \otimes_{c} E_{2}\right)^{\wedge}$ defined in Proposition 5.4 is onto, then $E_{1}$ or $E_{2}$ is of type $\mathrm{I}$.

Proof. Suppose $E_{i}$ is separable and that $S_{i}=\left\{x_{n}^{i}\right\}_{n=1}^{\infty}$ is a dense subset; then $\left\{\varepsilon_{i}\left(x_{n}^{i}\right)\right\}$ is dense in $C^{*}\left(E_{i}\right)$ : given $t_{i} \in C^{*}\left(E_{i}\right)$ and $\varepsilon>0$ there is $s_{i} \in E_{i}$ so that $\left|t_{i}-\varepsilon_{i}\left(s_{i}\right)\right|<\varepsilon / 2$ and there is $x_{n_{i}}^{i} \in S_{i}$ so that $\left|s_{i}-x_{n_{i}}^{i}\right|<\varepsilon / 2$. Then

$$
\begin{aligned}
\left|t_{i}-\varepsilon_{i}\left(x_{n_{i}}^{i}\right)\right| & \leqq\left|t_{i}-\varepsilon_{i}\left(s_{i}\right)\right|+\left|\varepsilon_{i}\left(s_{i}\right)-\varepsilon_{i}\left(x_{n_{i}}^{i}\right)\right| \\
& \leqq\left|t_{i}-\varepsilon_{i}\left(s_{i}\right)\right|+\left|s_{i}-x_{n_{i}^{i}}\right|<\varepsilon .
\end{aligned}
$$

Evidently, the fact that $G$ is onto implies that $G_{1}:\left[C^{*}\left(E_{1}\right)\right]^{\wedge} \times\left[C^{*}\left(E_{2}\right)\right]^{\wedge} \rightarrow$ $\left[C^{*}\left(E_{1} \otimes_{c} E_{2}\right)\right]^{\wedge}=\left[C^{*}\left(E_{1}\right) \otimes_{\nu} C^{*}\left(E_{1}\right)\right]^{\wedge}$ is onto. From Theorem 2 [17] we conclude that $C^{*}\left(E_{1}\right)$ or $C^{*}\left(E_{2}\right)$ is of type I and hence that $E_{1}$ or $E_{2}$ is of type I. This completes the proof.

\section{6. *-semisimplicity.}

6.1. In this section we consider the problem of whether $*$-semisimplicity (defined below) of $E_{1}, E_{2}$ is passed on to $E_{1} \otimes_{c} E_{2}$ for a suitable norm $c$. This problem appears quite difficult and we present only a partial solution in the form of a necessary and sufficient condition that a tensor product of $*$-semisimple Banach-*algebras be $*$-semisimple. This condition is interesting in so far as it indicates a similarity between $*$-semisimplicity and the more general concepts semisimplicity and strong semisimplicity (for definitions, see [18, p. 55 and 59]): Gelbaum [6] and Gil de Lamadrid [8] show that if $A$ and $B$ are commutative semisimple Banach algebras and $c$ is an ordinary norm so that $A \otimes_{c} B$ is a Banach algebra then $A \otimes_{c} B$ is semisimple iff the natural mapping of $A \otimes_{c} B$ into $A \otimes_{\lambda} B$ (to be defined) is $1-1$. The same result holds for $\gamma=c$ and strong semisimplicity replacing semisimplicity when only one of the algebras is assumed commutative [26]. Here we 
show that the same condition connects *-semisimplicity of $E_{1}$ and $E_{2}$ and of $E_{1} \otimes_{c} E_{2}$ for a wide class of Banach-*-algebras.

6.2. Let $A$ be a $*$-algebra. The intersection of the kernels of all $*$-representations of $A$ on Hilbert space is called the $*$-radical $R^{*}$. If $R^{*}=\{0\}$ then $A$ is $*$-semisimple. This definition of $R^{*}$ is a slight extension of that given in 2.8 .

6.3. Suppose $E_{1}$ and $E_{2}$ are normed vector spaces and $c$ is an ordinary norm on $E_{1} \otimes E_{2}$, i.e. $\lambda \leqq c \leqq \gamma$. Since $\lambda \leqq c$ the identity mapping $E_{1} \otimes E_{2} \rightarrow E_{1} \otimes_{\lambda} E_{2}$ has a unique extension to $E_{1} \otimes_{c} E_{2}$, i.e. we can define

$$
\tau_{c}: E_{1} \otimes_{c} E_{2} \rightarrow E_{1} \otimes_{\lambda} E_{2} .
$$

$\tau_{c}$ is a homomorphism. If $E_{1}, E_{2}$ are normed algebras, then $\tau_{c}$ is an algebra homomorphism. If $\tau_{c}$ is an isomorphism we also say that $c$ is a faithful norm.

6.4. The main result of this section is that faithfulness of $c$ is necessary and sufficient for the tensor product of $*$-semisimple Banach-*-algebras $E_{1}, E_{2}$ to be *-semisimple, at least when $E_{1}$ or $E_{2}$ belongs to a rather wide class of algebras. This class is the class of Banach-*-algebras for which $C^{*}(E)$ satisfies Takesaki's condition $(T)$ [23]. As mentioned in $3.5 C^{*}(E)$ satisfies condition $(T)$ iff $C^{*}(E) \otimes_{\nu} B$ $=C^{*}(E) \otimes_{\alpha} B$ for any $C^{*}$-algebra $B$. We say $E$ satisfies condition $(T)$ if $C^{*}(E)$ does.

For the proof a few preliminary facts are needed. The proof of the first of these is immediate from the definition of the enveloping algebra and will be omitted.

LEMMA 6.5. If $E$ is a Banach-*-algebra with enveloping algebra $C^{*}(E)$, then $E$ is *-semisimple iff the enveloping mapping $\varepsilon: E \rightarrow C^{*}(E)$ is 1-1.

LeMma 6.6. $\alpha$ is faithful; i.e. if $E$ and $F$ are $C^{*}$-algebras, then $\tau_{\alpha}: E \otimes_{\alpha} F \rightarrow E \otimes_{\lambda} F$ is $1-1$.

Proof. Suppose $\tau_{\alpha}(t)=0$; from the definition of the $\lambda$-norm it follows that $x^{\prime} \otimes y^{\prime}(t)=0$ for any $x^{\prime} \in E_{*}$ and $y^{\prime} \in F_{*}$. By the definition of $\alpha$ we can identify $E \otimes_{\alpha} F$ with a sub-*-algebra of $L\left(H_{1} \otimes_{\sigma} H_{2}\right)$ for suitable Hilbert spaces $H_{1}$ and $H_{2}$. To show that $t=0$ it suffices to show that $\left(t h_{1} \mid h_{2}\right)=0$ for any $h_{1}, h_{2} \in H_{1} \otimes_{\sigma} H_{2}$ where $(\cdot \mid \cdot)$ denotes the inner product; in fact, it suffices to consider $h_{1}, h_{2}$ $\in H_{1} \otimes H_{2}$ and hence, since $(\cdot \mid \cdot)$ is linear in both entries, to show that

$$
\left(t\left(x_{1} \otimes y_{1}\right) \mid\left(x_{2} \otimes y_{2}\right)\right) \text { for any } x_{1}, x_{2} \in H_{1}, y_{1}, y_{2} \in H_{2} \text {. }
$$

Pick $x_{1}, x_{2} \in H_{1}$ and $y_{1}, y_{2} \in H_{2} . f(\cdot)=\left(\cdot x_{1} \mid x_{2}\right)$ defines a continuous linear functional on $L\left(H_{1}\right)$ and hence on $E$; likewise, $g(\cdot)=\left(\cdot y_{1} \mid y_{2}\right)$ is a continuous linear functional on $F$. An easy computation will show that

$$
\left(t\left(x_{1} \otimes y_{1}\right) \mid\left(x_{2} \otimes y_{2}\right)\right)=f \otimes g(t)=0 .
$$

This proves the lemma.

REMARK 6.7. A similar result does not hold for $\nu$. For if $\tau_{v}: E \otimes_{\nu} F \rightarrow E \otimes_{\lambda} F$ is $1-1$ then $\tau_{\nu \alpha}: E \otimes_{\nu} F \rightarrow E \otimes_{\alpha} F$ is $1-1$ (since $\tau_{v}=\tau_{\alpha} \circ \tau_{v \alpha}$ ); and since $E \otimes_{\nu} F$ and 
$E \otimes_{\alpha} F$ are $C^{*}$-algebras this implies that $\nu=\alpha$. This, however, is not generally true (see [23]). It is this fact that forces us to limit ourselves to Banach-*-algebras satisfying condition $(T)$.

6.8. Since $\varepsilon: E \rightarrow C^{*}(E)$ is a bounded linear mapping it defines a dual mapping $\varepsilon_{*}: C^{*}(E)_{*} \rightarrow E_{*}$. In fact, if $a^{\prime} \in C^{*}(E)_{*}$, then $\varepsilon_{*}\left(a^{\prime}\right)=f_{a^{\prime}} \in E_{*}$ is defined by $f_{a^{\prime}}(x)=a^{\prime}(\varepsilon(x))$ for all $x \in E$.

We are now ready to state and prove

THEOREM 6.9. Suppose $E_{1}$ and $E_{2}$ are Banach-*-algebras with approximate identities and that one of them satisfies condition $(T)$. If $c$ is an ordinary norm compatible with multiplication and involution on $E_{1} \otimes E_{2}$ then $E_{1} \otimes_{c} E_{2}$ is *-semisimple iff $c$ is faithful.

Proof. Suppose first that $E_{1} \otimes_{c} E_{2}$ is $*$-semisimple. Choose $t_{0} \in E_{1} \otimes_{c} E_{2}$ such that $\tau_{c}\left(t_{0}\right)=0$ where $\tau_{c}: E_{1} \otimes_{c} E_{2} \rightarrow E_{1} \otimes_{\lambda} E_{2}$ is the canonical mapping (6.3). Let $\tau_{\alpha}$ denote the canonical mapping of $C^{*}\left(E_{1}\right) \otimes_{\alpha} C^{*}\left(E_{2}\right)$ into $C^{*}\left(E_{1}\right) \otimes_{\lambda} C^{*}\left(E_{2}\right)$. Then by Lemma $6.6 \alpha$ is faithful.

Now, $\tau_{c}\left(t_{0}\right)=0 \Rightarrow x^{\prime} \otimes y^{\prime}\left(t_{0}\right)=0$ for any $x^{\prime} \in E_{1 *}, y^{\prime} \in E_{2 *}$; in particular,

$$
\left[\left(\varepsilon_{E_{1}}\right)_{*}\left(a^{\prime}\right) \otimes\left(\varepsilon_{E_{2}}\right)_{*}\left(b^{\prime}\right)\right]\left(t_{0}\right)=0
$$

for any $a^{\prime} \in C^{*}\left(E_{1}\right)_{*}, b^{\prime} \in C^{*}\left(E_{2}\right)_{*}$ (see 6.8). From (6.8) and (6.10) it follows that

$$
\left(\left(a^{\prime} \circ \varepsilon_{E_{1}}\right) \otimes\left(b^{\prime} \circ \varepsilon_{E_{2}}\right)\right)\left(t_{0}\right)=0
$$

for any $a^{\prime} \in C^{*}\left(E_{1}\right)_{*}, b^{\prime} \in C^{*}\left(E_{2}\right)_{*}$ and hence that $a^{\prime} \otimes b^{\prime}\left(\varepsilon_{E_{1}} \otimes \varepsilon_{E_{2}}\right)\left(t_{0}\right)=0$ for any $a^{\prime} \in C^{*}\left(E_{1}\right)_{*}, b^{\prime} \in C^{*}\left(E_{2}\right)_{*}$. Since $E_{1}$ or $E_{2}$ satisfies condition $(T)$, the proof of Corollary 4.9 enables us to conclude that $\lambda\left(\tau_{\alpha}\left(\varepsilon\left(t_{0}\right)\right)\right)=0$. By the above this implies that $\varepsilon\left(t_{0}\right)=0$ and hence (Lemma 6.5) that $t_{0}=0$ i.e. $\tau_{c}$ is $1-1$.

Conversely, suppose $c$ is faithful and that $s_{0} \in E_{1} \otimes_{c} E_{2}$ is chosen so that $s_{0} \neq 0$; then $\tau_{c}\left(s_{0}\right)=t_{0} \neq 0$. As is known [19, I-III] any element $t$ in $E_{1} \otimes_{\lambda} E_{2}$ defines a continuous linear transformation $T_{t}: E_{1 *} \rightarrow E_{2}$ and also $S_{t}: E_{2 *} \rightarrow E_{1}$.

If $t$ is a finite tensor in $E_{1} \otimes_{\lambda} E_{2}$ and $\sum x_{i} \otimes y_{i}$ is a representative of $t$, then

$$
T_{t}\left(x^{\prime}\right)=\sum x^{\prime}\left(x_{i}\right) y_{i} \text { for all } x^{\prime} \in E_{1 *} .
$$

This defines $T_{t}$ for every $t$ in a dense subspace of $E_{1} \otimes_{\lambda} E_{2}$. The definition is completed by use of the customary argument of extension by continuity. $S_{t}$ is defined similarly.

Consider $T_{t_{0}}$ and $S_{t_{0}} \cdot t_{0} \neq 0 \Rightarrow T_{t_{0}} \neq 0 \Rightarrow$ there is $x_{0}^{\prime} \in E_{1 *}$ such that $0 \neq T_{t_{0}}\left(x_{0}^{\prime}\right)$ $\in E_{2}$. Since $E_{2}$ is $*$-semisimple, $\varepsilon_{E_{2}}\left(T_{t_{0}}\left(x_{0}^{\prime}\right)\right) \neq 0$ (Lemma 6.5); hence there is $b_{0}^{\prime} \in C^{*}\left(E_{2}\right)_{*}$ such that

$$
b_{0}^{\prime}\left(\varepsilon_{E_{2}}\left(T_{t_{0}}\left(x_{0}^{\prime}\right)\right)\right) \neq 0 .
$$

Let $f_{b_{0}^{\prime}}=\left(\varepsilon_{E_{2}}\right)_{*}\left(b_{0}^{\prime}\right)$ (see 6.8). Now consider $S_{t_{0}}: E_{2 *} \rightarrow E_{1} . S_{t_{0}}$ is not zero; in fact, it is easy to see that

$$
x_{0}^{\prime}\left(S_{t_{0}}\left(f_{b_{0}^{\prime}}\right)\right)=b_{0}^{\prime}\left(\varepsilon_{E_{2}}\left(T_{t_{0}}\left(x_{0}^{\prime}\right)\right)\right) \neq 0
$$


where $x_{0}^{\prime}$ is the functional chosen above. From the $*$-semisimplicity of $E_{1}$ it follows that there is $a_{0}^{\prime} \in C^{*}\left(E_{1}\right)_{*}$ such that

$$
a_{0}^{\prime}\left(\varepsilon_{E_{1}}\left(S_{t_{0}}\left(f_{b_{0}^{\prime}}\right)\right)\right) \neq 0 .
$$

But a straightforward calculation will show that the left-hand side of (6.11) equals

$$
\left(a_{0}^{\prime} \otimes b_{0}^{\prime}\right)\left(\varepsilon_{E_{1}} \otimes \varepsilon_{E_{2}}\left(s_{0}\right)\right),
$$

thus showing that $\varepsilon_{E_{1}} \otimes \varepsilon_{E_{2}}\left(s_{0}\right) \neq 0$ and therefore $\varepsilon\left(s_{0}\right) \neq 0$. We have shown that $\varepsilon$ is $1-1$ and consequently $(6.5)$ that $E_{1} \otimes_{c} E_{2}$ is $*$-semisimple.

The following converse result holds

Proposition 6.10. If $E_{1}, E_{2}$ are Banach-*-algebras with approximate identities and $c$ an ordinary norm such that $E_{1} \otimes_{c} E_{2}$ is a Banach-*-algebra, then $E_{1}$ and $E_{2}$ are *-semisimple if $E_{1} \otimes_{c} E_{2}$ is *-semisimple.

Proof. Let $0 \neq x_{i} \in E_{i}$; since $c$ is a crossnorm $x_{1} \otimes x_{2} \in E_{1} \otimes_{c} E_{2}$ is not zero. Therefore there is a representation $\pi$ of $E_{1} \otimes_{c} E_{2}$ such that $\pi\left(x_{1} \otimes x_{2}\right) \neq 0$. Letting $\pi_{1}$ and $\pi_{2}$ denote the restrictions of $\pi$ to $E_{1}$ and $E_{2}$ respectively, and using Lemma 4.7 we conclude that since $\pi_{1}\left(x_{1}\right) \pi_{2}\left(x_{2}\right)=\pi\left(x_{1} \otimes x_{2}\right), \pi_{1}\left(x_{1}\right) \neq 0$ and $\pi_{2}\left(x_{2}\right) \neq 0$. It follows that $E_{1}$ and $E_{2}$ are $*$-semisimple.

Grothendieck [14, p. 90] has shown that if $G$ is a locally compact Hausdorff space and $B$ is a Banach space, then the space of all continuous functions vanishing at infinity from $G$ into $B, C(G, B)$ with the sup norm is isometrically isomorphic to $C(G) \otimes_{\lambda} B$ where $C(G)$ denotes the space of $C$-valued continuous functions vanishing at infinity. It is not difficult to see that this identification also preserves the natural multiplications if $B$-and hence $C(G, B)$-is a Banach algebra. Moreover, if $B$ is a Banach-*-algebra, then the above isometry can easily be shown to preserve the natural involutions.

On the basis of these remarks we can state the following corollary, the proof of which is obvious from the above and Theorem 6.9. (It should be noted that commutative algebras satisfy condition $(T)$ [23, Theorem 1].)

Corollary 6.11. Let $G$ be a locally compact $T_{2}$ space and $B$ a Banach-*-algebra with approximate identity. Let $C(G, B)$ be the algebra of all continuous functions from $G$ to $B$ vanishing at infinity with the sup norm and involution $f^{*}(g)=(f(g))^{*}$ for any $f \in C(G, B) . C(G, B)$ is *-semisimple if and only if $B$ is *-semisimple.

Proof. The proof is clear from the above, since $C(G)$ has an approximate identity.

Corollary 6.10 can be proved directly. We have included it here primarily to introduce the identification $C(G, B)=C(G) \otimes_{\lambda} B$. This identification is also valid when $C(G)$ is not commutative (e.g. when we use convolution multiplication) and will be used again in $\$ 7$. 
7. Examples. In this section we apply the above theory to vector-valued group algebras over a compact $T_{2}$ group $G$. But first let us consider the numerical-valued case.

7.1. Complex-valued group algebras. Let $G$ be a compact topological group in which the topology is Hausdorff and let $m$ denote the Haar measure on $G$ (since $G$ is compact, $m$ is left-as well as right-invariant, i.e. $G$ is unimodular). We normalize $m$ so that $m(G)=1$.

Kaplansky [12] shows that if $1 \leqq p<\infty$ then $L^{p}(G)$, the space of $C$-valued functions $f$ on $G$ for which

$$
|f|_{p}=\left[\int|f(g)|^{p} d m(g)\right]^{1 / p}<\infty,
$$

is a semisimple dual Banach-*-algebra. Multiplication in $L^{p}(G)$ is defined by convolution, i.e. if $f_{1}, f_{2} \in L^{p}(G)$, then $f_{1} * f_{2}$ is defined by

$$
\left(f_{1} * f_{2}\right)(g)=\int f_{1}\left(g g_{1}\right) f_{2}\left(g_{1}^{-1}\right) d m\left(g_{1}\right)
$$

Involution is defined by

$$
f^{*}(g)=f\left(g^{-1}\right)^{*} \text { for } f \in G
$$

where the latter * denotes complex conjugation.

We shall show that

$$
C^{*}\left(L^{p_{1}}(G)\right)=C^{*}\left(L^{p_{2}}(G)\right), \quad p_{1}, p_{2} \in[1, \infty) .
$$

This will be accomplished by showing that

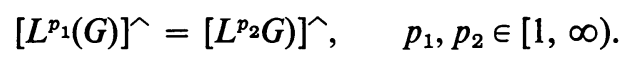

(7.3) is to be interpreted as a symbolic expression of the existence of a particular 1-1 correspondence onto. We shall return to this point shortly. First, it is well known that the compactness of $G$ implies that if $\infty>p \geqq q \geqq 1$, then

$$
L^{p}(G) \subset L^{q}(G) ;
$$

in fact, $L^{p}(G)$ is an ideal in $L^{q}(G)$. Moreover, if $C^{c}(G)$ denotes the algebra of all continuous complex-valued functions on $G$ with sup-norm, convolution multiplication and involution defined by (7.2) then

$$
C^{c}(G) \subset L^{p}(G), \quad \infty>p \geqq 1
$$

as an ideal. We denote the norm in $L^{p}(G)$ by $|\cdot|_{p}$ and in $C^{c}(G)$ by $|\cdot|_{\infty}$ and have, since the measure is normalized on $G$, that

$$
|\cdot|_{\infty} \geqq|\cdot|_{p} \geqq|\cdot|_{1} \quad \text { for } p \in[1, \infty)
$$

$[15$, p. 430]. 
Because of the inclusions (7.4) and (7.5) and the norm relationship (7.6) it is clear that

$$
\left[L^{1}(G)\right]^{\wedge} \subset\left[L^{p}(G)\right]^{\wedge} \subset\left[C^{c}(G)\right]^{\wedge}
$$

for any $p, 1 \leqq p<\infty$ in the sense that a topologically irreducible representation of $L^{1}(G)$ uniquely determines (by restriction) a topologically irreducible representation of $L^{p}(G)(1 \leqq p<\infty)$ which in turn uniquely determines (again by restriction) a topologically irreducible representation of $C^{c}(G)$. Consequently (7.3) can be proved by showing that

$$
\left[C^{c}(G)\right]^{\wedge}=\left[L^{1}(G)\right]^{\wedge}
$$

Again the equality means the existence of a special 1-1 correspondence onto; any topologically irreducible representation of $L^{1}(G)$ defines by restriction a topologically irreducible representation of $C^{c}(G)$; conversely, a topologically irreducible representation of $C^{c}(G)$ is (uniquely) extendible to a topologically irreducible representation of $L^{1}(G)$. We give an indication of the proof of this.

Since any unitary representation of $G$ generates a representation of $L^{1}(G)$ [15, pp. 378-379], to show the validity of (7.7), it suffices to prove that if $\bar{\pi} \in\left[C^{c}(G)\right]^{\wedge}$ then $\pi \in \bar{\pi}$ generates a unitary representation of $G$. In [15, pp. 377-378] it is proved that any topologically irreducible representation of $L^{1}(G)$ induces a unitary representation of $G$. The same proof works to show that a topologically irreducible representation of $C^{c}(G)$ generates a unitary representation of $G$. For this reason, the proof will not be reproduced here. Because of these identifications we shall use $\hat{G}$ to denote the spectrum of $L^{p}(G), 1 \leqq p<\infty$ and the same symbol for $\left[C^{c}(G)\right]^{\wedge}$.

We can now complete the proof of the equation (7.3). According to the above discussion it suffices to prove that $C^{*}\left(L^{1}(G)\right)=C^{*}\left(C^{c}(G)\right)$. Since $C^{c}(G)$ is $*$-semisimple $C^{*}\left(C^{c}(G)\right)$ can be interpreted as the completion of $C^{c}(G)$ with respect to the norm $|f|^{\prime}=\sup _{\bar{\pi} \in\left[C^{c}(G)\right]_{\wedge}}|\pi(f)|(2.8)$. In the same vein $C^{*}\left(L^{1}(G)\right)$ is the completion of $L^{1}(G)$ with respect to the norm $|f|^{\prime \prime}=\sup _{\bar{\pi} \in\left[L^{1}(G)\right]^{\wedge}}|\pi(f)|$. It follows from (7.7) that $|f|^{\prime}=|f|^{\prime \prime}$ for $f \in C^{c}(G)$. Since $C^{c}(G)$ is dense in $L^{1}(G)$ with respect to the 1-norm we can conclude that $C^{*}\left(C^{c}(G)\right)=C^{*}\left(L^{1}(G)\right)$. We have proved

Proposition 7.8. $C^{*}\left(L^{p_{1}}(G)\right)=C^{*}\left(L^{p_{2}}(G)\right)$ for any $p_{1}, p_{2} \in[1, \infty)$.

On the basis of Proposition 7.8 we shall denote the enveloping algebra of $L^{1}(G)$ by $C^{*}(G)$.

Finally, we observe that $L^{p}(G), 1 \leqq p<\infty$ is of type $\mathrm{I}$, since $C^{*}(G)$ is of type $\mathrm{I}$. A proof of the fact that $C^{*}(G)$ is of type I can be found in $[3,15.1 .5,4.2 .4$ and 5.5.2]. Since $L^{p}(G)$ is known to have approximate identities the theory developed in $\S \S 4,5$ and 6 applies to tensor products involving $L^{p}(G)$.

7.9. Vector-valued group algebras. Examples of such tensor products are provided by the spaces $B^{p}(G, A)$ where $G$ is a compact Hausdorff group, $A$ a Banach 
algebra or Banach-*-algebra and $1 \leqq p<\infty$. These spaces have been studied by Spicer [20], [21] and are defined as the spaces of functions $f: G \rightarrow A$ for which

$$
\left[\int|f(g)|^{p} d m(g)\right]^{1 / p}<\infty .
$$

$B^{p}(G, A)$ is normed by defining

$$
|f|_{p}=\left[\int|f(g)|^{p} d m(g)\right]^{1 / p}
$$

Spicer [20, Chapter III, Theorem 1] shows that $B^{p}(G, A)=L^{p}(G) \otimes_{p} A$ where the norm on the tensor product is defined in the following fashion.

If $t=\sum x_{i} \otimes y_{i}$ is a finite tensor in $L^{p}(G) \otimes A$ consider the associated expression $\sum x_{i}(\cdot) y_{i}$ which defines a function from $G$ into $A$. We define

$$
p(t)=\left[\int\left|\sum x_{i}(g) y_{i}\right|^{p} d m(g)\right]^{1 / p} .
$$

This norm is shown [20, Chapter III, Proposition 3] to be an ordinary norm and compatible with multiplication. It is also compatible with involution.

With $t$ as before

$$
\begin{aligned}
p\left(t^{*}\right) & =\left[\int\left|\sum x_{i}^{*}(g) y_{i}^{*}\right|^{p} d m(g)\right]^{1 / p} \\
& =\left[\int\left|\sum \overline{x_{i}\left(g^{-1}\right)} y_{i}^{*}\right|^{p} d m(g)\right]^{1 / p} \\
& =\left[\int \mid\left(\left.\left.\sum x_{i}\left(g^{-1}\right) y_{i}\right|^{*}\right|^{p} d m(g)\right]^{1 / p}\right. \\
& =\left[\int\left|\sum x_{i}\left(g^{-1}\right) y_{i}\right|^{p} d m(g)\right]^{1 / p} \\
& =\left[\int\left|\sum x_{i}(g) y_{i}\right|^{p} d m(g)\right]^{1 / p}=p(t) .
\end{aligned}
$$

Since the involution is isometric with respect to $p$ on $L^{p}(G) \otimes^{p} A$, the same is the case on $L^{p}(G) \otimes_{p} A$.

If $A$ is a Banach-*-algebra with approximate identity then $\$ \$ 4$ and 5 immediately give us the following structure theorems:

Proposition 7.10. $C^{*}\left(B^{p}(G, A)\right)=C^{*}(G) \otimes_{\alpha} C^{*}(A)$ and

Proposition 7.11. $\left[B^{p}(G, A)\right]^{\wedge}=\hat{G} \times \hat{A}$.

Finally, we prove

Proposition 7.12. If $A$ is a Banach-*-algebra with approximate identity and $G$ is a compact $T_{2}$ group, then $B^{p}(G, A)$ is *-semisimple iff $A$ is $*$-semisimple $[1 \leqq p<\infty]$. 
Proof. The proof makes use of an extension of Grothendieck's result concerning spaces of vector valued continuous functions. Spicer [20, pp. 38-39] observes that this result also implies that $C^{c}(G, A)=C^{c}(G) \otimes_{\lambda} A$.

First we note that Theorem 6.9 and Proposition 6.10 do not apply directly to the present situation since $C^{c}(G)$ does not have an approximate identity. However, an examination of the relevant parts of the proof of Theorem 6.9 shows that if we assume the cross norm $c$ to be faithful then it follows that $\varepsilon_{E_{1}} \otimes \varepsilon_{E_{2}}$ is 1-1 and therefore that $\varepsilon$ is 1-1 i.e. that $E_{1} \otimes_{c} E_{2}$ is $*$-semisimple provided that $E_{1}$ and $E_{2}$ are (we are using the notation of Theorem 6.9). It follows from these observations that $C^{c}(G, A)$ is $*$-semisimple if $A$ is *-semisimple.

Suppose $A$ is $*$-semisimple. Then $C^{c}(G, A)$ is $*$-semisimple. We want to show that $B^{p}(G, A)$ is $*$-semisimple. Let $t \in B^{p}(G, A)$ and suppose $\pi(t)=0$ for all

$$
\bar{\pi} \in\left[B^{p}(G, A)\right]^{\wedge}=\left[C^{c}(G, A)\right]^{\wedge} .
$$

By Proposition 3 of Chapter II in [20] we can choose a net $\left\{w_{v}\right\} \subset C^{c}(G, A)$ such that $\left|w_{v}\right|_{1} \leqq 1$ and $\left\{w_{v}\right\}$ otherwise acts as an approximate identity in $B^{p}(G, A)$. Since $C^{c}(G, A)$ is an ideal in $B^{p}(G, A)$ [20, p. 15] it follows that $w_{v} * t \in C^{c}(G, A)$ for all $\nu$. Moreover $\pi(t)=0 \Rightarrow \pi\left(w_{v} * t\right)=0$ for any $\bar{\pi} \in\left[C^{c}(G, A)\right]^{\wedge}$ and for all $\nu$. Since $w_{v} * t \rightarrow t$ and since the $*$-semisimplicity of $C^{c}(G, A)$ implies that $w_{v} * t=0$ for all $\nu$, it follows that $t=0$, i.e. $B^{p}(G, A)$ is $*$-semisimple.

The other direction follows directly from the fact that $A$ can be identified with a closed ideal in $C^{c}(G, A)$ (the constant functions).

\section{BIBLIOGRAPHY}

1. N. Bourbaki, Algèbre multilinéaire, Hermann, Paris, 1948.

2. J. Dixmier, Les algèbrés d'opérateurs dans l'espace hilbertien, Gauthier-Villars, Paris, 1957.

3. - Les $C^{*}$-algèbres et leur représentations, Gauthier-Villars, Paris, 1964.

4. B. Gelbaum, Tensor products of Banach algebras, Canad. J. Math. 11 (1959), 297-310.

5. - Note on the tensor product of Banach algebras, Proc. Amer. Math. Soc. 12 (1961), 750-757.

6. - Tensor products and related questions, Trans. Amer. Math. Soc. 103 (1962), $525-548$.

7. J. Gil de Lamadrid, Uniform cross norms and tensor products of Banach algebras, Bull. Amer. Math. Soc. 69 (1963), 797-803.

8. - Uniform cross norms and tensor products of Banach algebras, Duke Math. J. 32 (1965), 359-368.

9. A. Grothendieck, Produits tensoriels topologiques et espaces nucléaires, Mem. Amer. Math. Soc. No. 16, 1955.

10. A. Guichardet, Caractères et représentations des produits tensoriels de $C^{*}$-algèbres, Ann. Sci. École Norm. Sup. 81 (1964), 189-206.

11. - Tensor product of $C^{*}$-algebras, Soviet Math. Dokl. 6 (1965), 110-213.

12. I. Kaplansky, Dual rings, Ann. of Math. 49 (1948), 689-701.

13. J. L. Kelley, General topology, Van Nostrand, Princeton, N. J., 1955.

14. L. H. Loomis, An introduction to abstract harmonic analysis, Van Nostrand, Princeton, N. J., 1953. 
15. M. Naimark, Normed rings, rev. ed., Noordhoff, Groningen, 1964.

16. K. Okayasu, On the tensor products of $C^{*}$-algebras, Tôhoku Math. J. 18 (1966), 325-331.

17. T. Okayasu and M. Takesaki, Dual spaces of tensor products of $C^{*}$-algebras, Tôhoku Math. J. 18 (1966), 332-337.

18. C. E. Rickart, General theory of Banach algebras, Van Nostrand, Princeton, N. J., 1960.

19. R. Schatten, $A$ theory of cross spaces, Princeton Univ. Press, Princeton, N. J., 1950.

20. D. Spicer, Group algebras of vector-valued functions, Thesis, Univ. of Minnesota, Minneapolis, 1965.

21. - Semisimplicity of group algebras of vector-valued functions, Proc. Amer. Math. Soc. 19 (1968), 573-577.

22. M. Takesaki, A note on the cross-norm of the direct product of operator algebra, Kōdai Math. Sem. Rep. 10 (1958), 137-140.

23. - On the cross norm of the direct product of $C^{*}$-algebras, Tôhoku Math. J. 16 (1964), 111-122.

24. T. Turumaru, On the direct product of operator algebra. I. Tôhoku Math. J. 4 (1952), 242-251.

25. A. Wulfsohn, Produit tensoriel de $C^{*}$-algèbres, Bull. Sci. Math. 87 (1963), 13-27.

26. K. B. Laursen, Tensor products of Banach-*-algebras, Thesis, Univ. of Minnesota, Minneapolis, 1967.

UNIVERSITY OF MINNESOTA,

MinNEAPOLIS, MiNNESOTA

UNIVERSITY OF AARHUS,

Aarhus, Denmark 